\title{
Autoantibody profiles linked to ethnicity in pediatric SLE
}

In patients with pediatric systemic lupus erythematosis (SLE), the prevalence of several autoantibodies varies with ethnic background and can be used to identify groups of patients with similar clinical characteristics, according to a study published in The Journal of Rheumatology. "We have shown that ethnicity is a very important determinant in autoantibody production and clinical phenotype, and that our pediatric cohort differs from previously described adult cohorts," says Earl Silverman, one of the study's investigators.

\section{Classifying the patients ... according to autoantibody profiles identified three distinct 'clusters' 77}

Taking into account the ethnic background of the 156 patients in this cohortall of whom were diagnosed with SLE before the age of 18 years - the prevalence of antiU1RNP and anti-Sm antibodies was significantly higher in non-white than in white patients, confirming earlier observations in adults. The frequencies of the other eight autoantibodies tested, however, including antibodies against SSA (Ro), SSB (La) and double-stranded (ds)DNA, did not differ significantly between ethnic groups.

Classifying the patients in this pediatric cohort according to autoantibody profiles identified three distinct 'clusters', with each one displaying characteristic clinical and laboratory features of SLE. This cluster analysis approach enabled the investigators to examine how multiple factors interacted to form these groups, and to generate hypotheses about correlations between autoantibody prevalence and clinical characteristics. For instance, cluster 1 , which had the highest proportion of white patients, was characterized by a high prevalence of anti-dsDNA antibodies only, and was associated with an overall mild disease course over the 8-year study period. By contrast, cluster 2 (characterized by a high frequency of dsDNA and eight other antibodies) was most often associated with renal failure and anemia, and cluster 3 with neuropsychiatric disease.

As the study was conducted at a single center, whether the differences in autoantibody profiles are generalizable to other pediatric cohorts remains to be seen. If confirmed, however, the association between autoantibody clusters and clinical phenotype could be important for predicting long-term disease outcome for patients with this disease.

\section{Sarah Price}

Original article Jurencák, R. et al. Autoantibodies in pediatric systemic lupus erythematosus: ethnic grouping, cluster analysis and clinical correlations. J. Rheumatol. 36, 416-421 (2009). 